\title{
Zwischen Entgrenzung und Re-Nationalisierung. Die Ambivalenz religiöser Überzeugungen in der postsäkularen Öffentlichkeit europäischer Gesellschaften
}

\author{
Martin Breul
}

This text analyses the potentials and dangers of a publicly present religion in a postsecular society. On the one hand, religions offer essential resources of motivation to engage in public policy and are critical voices in public discourse. On the other hand, they are at constant risk of becoming instruments of exclusion and of collapsing into a nationalistic insularity. This ambivalence of religion can be overcome by identifying a narrow political sphere, in which the public justification of collectively binding norms takes place, and a broad sphere of civil society, which is open for a comprehensive orientation of society. This solution aims at acknowledging religious communities and individuals as important and highly relevant public agents while at the same time insisting on the liberality and neutrality of a pluralist constitutional state.

Martin Breul is Post-doc Fellow at the Department of Systematic Theology at the University of Cologne.

\section{Einleitung: Das Janusgesicht der Religion ${ }^{1}$}

In den letzten Jahrhunderten ist Europa zu einer zunehmend säkularen Region geworden, im globalen Vergleich wahrscheinlich sogar zur säkularsten Region weltweit. Während man, wie der Philosoph Charles Taylor bemerkt, in der frühen Neuzeit im Normalfall gläubig war und derjenige, der nicht glaubte, sich vor der Gesellschaft rechtfertigen musste, ist die Standardannahme in zeitgenössischen europäischen Gesellschaften die des säkularen Bürgers. Religiöser Glaube ist eine Option von vielen, und inzwischen steht derjenige, der ,noch“ an Gott glaubt, unter Rechtfertigungsdruck. ${ }^{2}$ Das säkulare Europa ist geprägt von einem bunten Mix aus Weltanschauungen, Wertvorstellungen und Religionen, die innerhalb des

1 Für wertvolle Hinweise zu einer früheren Version des Textes bedanke ich mich bei zwei anonymen Gutachtern von J-RaT.

2 Vgl. Taylor 2009, S. 14f. Taylor beschreibt dort einen „Wandel, der von einer Gesellschaft, in der es praktisch unmöglich war, nicht an Gott zu glauben, zu einer Gesellschaft führt, in der dieser Glaube auch für besonders religiöse Menschen nur eine menschliche Möglichkeit neben anderen ist.“(Vgl. Ebd., S. 16). 
säkularen Rahmens liberaler Rechtsstaaten mehr oder minder friedlich nebeneinander leben.

Dennoch ist der Prozess der Säkularisierung kein geradliniger Prozess der Privatisierung oder Ersetzung von Religionen durch vermeintlich vernünftigere Gegenstücke. Religion erweist sich auch in Europa als widerstandsfähig gegen Versuche, sich ihrer zu entledigen. Die Gleichung „Je mehr Modernisierung, desto weniger Religion" scheint eine falsche Prognose gewesen zu sein. Die Verabschiedung dieser Gleichung, die unter dem Namen „Säkularisierungsthese“ bekannt geworden ist, hat Jürgen Habermas dazu veranlasst, unsere zeitgenössische Gesellschaft als ,postsäkular“ zu bezeichnen. Sowohl soziologisch als auch philosophisch und theologisch sollte man sich demnach auf „das Fortbestehen religiöser Gemeinschaften in einer sich fortwährend säkularisierenden Gesellschaft " ${ }^{\text {3 }}$ einstellen. Wenn Religion also kein Auslaufmodell ist, stellt sich gerade für die politische Öffentlichkeit einer demokratischen Gesellschaft die Frage, welche Chancen und Potenziale, aber auch welche Gefahren sie birgt.

In meinem Text möchte ich mich darum der Frage widmen, welche Rolle Religion für die postsäkularen Gesellschaften eines zunehmend vereinten Europa im 21. Jahrhundert spielen kann. Ist sie gefährlich und darum maximal im privaten Bereich zu dulden, oder ist sie angesichts einer Identitätskrise europäischer Institutionen und Tendenzen zu einer Re-Nationalisierung eines der wenigen Bindemittel, welches nationale Grenzen überschreiten und damit Ressourcen europäischer Solidarität freisetzen kann?

Ich werde zunächst die Ambivalenz von Religionen, insbesondere wenn sie in der politischen Öffentlichkeit auftreten, herausstellen. Sie können nämlich zum einen als möglicherweise unabdingbare zivilgesellschaftliche Ressourcen, auf die ein entgrenztes und zusammenwachsendes Europa stärker angewiesen sein könnte als bisher angenommen, betrachtet werden (2), zum anderen aber auch als letztlich partikulare Lehren, die in pluralistischen Gesellschaften die Neutralität des Staates und die faire Behandlung von freien und gleichen Bürgern gefährden können (3) - eine Ambivalenz, die der Religionssoziologe José Casanova auch das „Janusgesicht der Religionen“4 nennt. Meine These ist, dass diesem Janusgesicht des Religiösen durchaus Rechnung getragen werden kann, indem eine Differenzierung verschiedener Kontexte der Rechtfertigung von Überzeugungen vorgenommen wird - es ist eine falsche Alternative, nur zwischen Religion als unabdingbarem einigenden Band der Gesellschaft und Religion als Instrument der Exklusion und Destabilisierung zu wählen (4). So können die rettungswürdigen und identitätsstiftenden Elemente aus Religionsgemeinschaften für eine vitale demokratische Öffentlichkeit nutzbar gemacht werden, ohne zugleich Fundamentalismen oder reaktionäre Abgrenzungsrhetorik durch einen kriterienlosen Inklusivismus mit an Bord zu holen.

3 Habermas 2001, S. 24.

4 Casanova 1994, S. 4. 


\section{Religion als Mittlerin der europäischen Einheit?}

Während Religion im ausgehenden 20. Jahrhundert aus philosophischer und soziologischer Warte eher als archaisches, vormodernes und zu überwindendes Element in der Geschichte der Menschheit betrachtet wurde, hat sich diese Einschätzung im beginnenden 21. Jahrhundert mehrheitlich gewandelt: Häufig wird auf die Potenziale, Ressourcen und Beiträge von Religionsgemeinschaften für öffentliche Diskurse und den Zusammenhalt der Gesellschaft verwiesen. Insbesondere in einem Europa, das zunehmend auf einem Weg der Ent-Grenzung und Vereinigung voranschreitet, könnte Religion wichtige Impulse liefern und vielleicht sogar das Projekt einer Einigung Europas vorantreiben.

Der oben bereits erwähnte Jürgen Habermas, der sich selbst in Anlehnung an Max Weber als „religiös unmusikalisch“ bezeichnet, ist einer der Hauptvertreter dieser Einschätzung. Für ihn gilt, dass angesichts einer „entgleisenden Modernisierung ${ }^{\text {"5 }}$ in einer postsäkularen Gesellschaft nach neuen Bündnispartnern gesucht werden muss, um den Zerwürfnissen und Pathologien der Moderne zu begegnen. Worin aber bestehen diese sogenannten Pathologien der Moderne, und inwiefern könnte Religion ein Heilmittel gegen diese sein?

Meiner Ansicht nach lassen sich die Potenziale der Religion für das Projekt Moderne (und damit auch für Europa) insbesondere an drei Beispielfällen zeigen, in denen gesellschaftliche Pathologien durchaus mit dem Behandlungsmittel Religion therapiert werden könnten: zum einen in der Frage nach der Motivation zu zivilgesellschaftlichem Engagement, zum anderen in der Frage nach Quellen nationenübergreifender, ,europäischer" Solidarität, und schließlich in der Frage nach der Bändigung eines entfesselten, im schlechtesten Sinne neoliberalen Kapitalismus. ${ }^{6}$

Das erste Beispiel für die Potenziale religiöser Überlieferungen ist das Problem der Motivation zu zivilgesellschaftlichem Engagement. Das Phänomen der Politikverdrossenheit, welches häufig zu einer zynischen oder resignativen Einstellung auf Seiten der Bürger führt, ist gerade für ein bisher kaum als kollektiver Akteur wahrgenommenes, vereintes Europa eine Gefahr. Nicht umsonst liegt die Wahlbeteiligung bei Europawahlen in nahezu allen europäischen Ländern niedriger als bei nationalen Wahlen. Zudem scheint es häufig ein ausgeprägtes Desinteresse an gesellschaftlichen Fragen zu geben, die die nationalen Grenzen übersteigen. Wer sich allerdings nicht nur als Adressat, sondern zugleich als Autor von Rechten und Gesetzen versteht, sollte an öffentlichen Diskursen und demokratischen Prozessen kommunikativ teilhaben und dabei nach Möglichkeit die eigenen Interessen mit einer Orientierung am Gemeinwohl in Einklang bringen. ${ }^{7}$ Diese Bereitschaft zu demokratischer Partizipation kann allerdings nicht vom

5 Habermas 2005, S. 247.

6 Vgl. zum Folgenden ausführlich Breul 2015, S. 105-144.

7 Vgl. Ebd., S. 109. 
Staat erzwungen, sondern „den Bürgern eines liberalen Gemeinwesens nur angesonnen werden" ${ }^{8}$ Mit anderen Worten: Das dauerhafte zivilgesellschaftliche und politische Engagement der Bürger, ohne dass der liberale Staat letztlich seine demokratische Identität aufgeben müsste, bedarf einer Motivation auf Seiten der Bürger, die weder zu einer Rechtspflicht gemacht noch aus dem Nichts heraus erschaffen werden kann. Es könnte sein, dass ausschließlich säkulare Motivationsquellen zu schwach sind, um zu zivilgesellschaftlichem Engagement, besonders auf der abstrakten europäischen Ebene, zu motivieren. Umfassende religiöse Überzeugungen stellen jedoch häufig motivationale Gründe dar, die zu einem erhöhten Engagement in öffentlichen Prozessen führen können. Vielleicht ist demnach gerade eine europäische Zivilgesellschaft also darauf angewiesen, die religiösen Überzeugungen einiger ihrer Bürger vorsichtig zu behandeln und diese nicht in einer aggressiv säkularistischen Manier in einen rein privaten Bereich abzudrängen.

Ein zweiter Bereich, in dem Religion mögliche Potenziale für ein sich entgrenzendes Europa darstellt, ist die für ein vereintes Europa notwendige Solidarität über die Grenzen von Nationalstaaten hinweg. Solidarität zeichnet sich im Gegensatz zu den unbedingten Pflichten moralischer oder rechtlicher Natur dadurch aus, dass sie nicht erzwingbare, über die minimalen unbedingten Pflichten hinausgehende Handlungen motiviert. ${ }^{9}$ Allerdings ist Solidarität schon innerhalb von großen Nationalstaaten ein sehr abstraktes und unpersönliches und damit rares Phänomen, welches rechtlich nicht erzwungen werden kann. Religionen können besondere Quellen der Solidarität sein, da sie zum einen die Einnahme eines universalistischen Standpunktes ermöglichen, der letztlich aus der achsenzeitlichen Umstellung von einer immanenten Mythologie auf Vorstellungen von Transzendenz resultiert. Wer es schafft, den Standpunkt des Alltäglichen und Innerweltlich-Schicksalhaften zu überschreiten, kann darum, in universalistischer Perspektive, die Bedürfnisse und Interessen der Mitbürger verstehen und trotz fehlender konkreter Bezüge an der Kritik an unsolidarischem Verhalten festhalten. Zum anderen bilden religiöse Überlieferungen dichte Narrative und symbolische Verkörperungen von Sinn ab, die Erfahrungen von Solidarität ermöglichen und damit wiederum Solidarität stiften können. Im Gegensatz zu rein vernünftigen Gründen sprechen Religionen durch gemeinsame Praktiken auch Leidenschaften, Emotionen und Sinne an, was zu einer semantisch reichhaltigeren Symbolsprache und damit zu möglichen Quellen von moralischer Motivation und Solidarität führen kann. ${ }^{10}$

Schließlich könnten, drittens, die Religionen Europas auch als Bündnispartner gegen einen entfesselten Kapitalismus in Anschlag gebracht werden. Eine hemmungslose globalisierte Ökonomie und die damit verbundene Verbreitung rein

8 Ebd., S. 110.

9 Vgl. zu dieser Begriffsanalyse auch Habermas 2013, S. 82-111.

10 Vgl. dazu auch Ders. 2012, S. 54-76. 
zweckrationalen Denkens führt dazu, dass ausschließlich Eigeninteressen verfolgende Egoisten sich lose zu einer Gesellschaft zusammenschließen, in der Bürger als ,vereinzelte, selbstinteressiert handelnde Monaden (...) nur noch ihre subjektiven Rechte wie Waffen gegeneinander richten" ${ }^{11}$ Dabei schreitet die Entsolidarisierung der Gesellschaft durch die Übertragung ökonomischer Imperative auf andere Lebensbereiche unaufhaltsam voran, wodurch rein strategische Erwägungen strukturell bevorteilt werden. Diese Entpolitisierung und Entsolidarisierung von Wirtschaft und Gesellschaft wird durch die Globalisierung noch verschärft, da sich durch sie die politischen Gestaltungsräume stetig reduzieren. Es ist darum eine offene Frage, ob sich eine deregulierte, globalisierte Marktökonomie überhaupt wieder so weit einfangen lassen kann, dass „der global entfesselte und verwilderte Kapitalismus noch gezähmt und in sozial verträgliche Bahnen gelenkt werden kann".${ }^{12}$ Dagegen erscheinen viele Religionen als Gegner der ungerechten Verteilung der Lasten der Globalisierung oder struktureller Benachteiligungen in einer rein auf instrumentelles Handeln und Nutzenmaximierung angelegten Weltgesellschaft. In Religionsgemeinschaften können „hinreichend differenzierte Ausdrucksmöglichkeiten und Sensibilitäten für verfehltes Leben, für gesellschaftliche Pathologien, für das Misslingen individueller Lebensentwürfe und die Deformation entstellter Lebenszusammenhänge “13 entdeckt werden, die im Bemühen um die Begrenzung der entgrenzten Ökonomie wertvolle Beiträge leisten und zu Mäßigung und Augenmaß anhalten können. Mit der Diagnose ökonomischer Entgleisungen trifft Habermas in meinen Augen einen entscheidenden Punkt, da jenseits einer rein formalen Diskursmoral Gemeinsinn, Solidarität und starke normative Bindungskräfte nötig sind, um eine zügellose Marktökonomie zu bändigen. Letztlich ist es ein Interesse des liberalen Verfassungsstaates selbst, alle (d.h. auch religiöse) Ursprünge der Solidarität und des Normbewusstseins der Bürger zu schützen. Damit überwindet Habermas zugleich den Paternalismus des klassischen Liberalismus, der religiöse Überzeugungen als vormoderne und für den politischen Diskurs eher lästige Überzeugungen betrachtete, mit denen man zwar möglichst zivilisiert umgehen sollte, von denen aber kein produktiver Beitrag für politische Problemlösungen zu erwarten war. $^{14}$

Diese drei Beispiele stellen mögliche Funktionen von Religionen aus politiktheoretischer Perspektive dar. Natürlich wäre es leicht, hier den Vorwurf der Instrumentalisierung bzw. Funktionalisierung zu erheben, so dass ich betonen möchte, dass es sich um einige wenige, vielleicht nicht einmal zentrale Rollen von Religion handelt. Auf die vielfältigen existenziellen, lebenspraktischen und innergemeinschaftlichen Dimensionen von Religion kann ich hier nicht näher

11 Ders. 2005, S. 112.

12 Ders. 2012, S. 109.

13 Ders. 2005, S. 115.

14 Vgl. dazu ausführlich Breul 2014, S. 57-67. 
eingehen; jedoch wäre in diesem Feld weitere begriffliche Arbeit hinsichtlich der Rolle und Relevanz religiöser Überzeugungen und Überlieferungen lohnenswert. $^{15}$

Zudem ist es wichtig zu betonen, dass die hier rekonstruierten, „postsäkularen" Versuche einer rettenden Aneignung religiöser Potenziale keinesfalls unkontrovers sind. So kritisiert beispielsweise Hans Joas, dass Habermas eine Art Etikettenschwindel betreibe, weil der Begriff der Postsäkularität impliziere, dass man eine säkulare Phase überwunden habe und sich nun auf den Fortbestand der Religion einrichte. Religion sei aber nie verschwunden, sondern in der zweiten Hälfte des 20. Jahrhunderts lediglich mit bedeutend weniger Aufmerksamkeit bedacht worden als derzeit. Darum gelte: ,Vielleicht wäre es besser gewesen, eine bisherige Unterschätzung selbstkritisch einzuräumen - statt sie mit einem Begriff zu verkleiden, der nach Epochenwandel klingt. “16 Ähnlich bestehen viele Religionssoziologen wie z. B. José Casanova, David Martin oder Karl Gabriel darauf, dass es einer genaueren, deskriptiven Studie von Einzelfällen bedürfe, um eine Großthese wie die Diagnose einer „postsäkularen Gesellschaft“ und die Notwendigkeit einer rettenden Aneignung der Potenziale der Religion zu postulieren. ${ }^{17}$ Diese kritischen Einwände gegen eine abstrakt-postsäkulare Hinwendung zu den Potenzialen der Religion können m. E. primär dazu dienen, ein entscheidendes Missverständnis zu beseitigen: Der Begriff der „Postsäkularität“ hat verschiedene Bedeutungsschichten, die von soziologisch-deskriptiven Zeitdiagnosen bis zu philosophisch-konzeptionellen bzw. normativen Analysen reichen. Die philosophisch-konzeptionellen Analysen der öffentlichen Potenziale von Religion sind dabei letztlich nicht von soziologischen Differenzen hinsichtlich einer neuen, postsäkularen Aufmerksamkeit für Religion abhängig - hier gilt es, die verschiedenen, sowohl philosophisch-normativen wie auch soziologisch-de-

15 Derzeit erfolgen eine Vielzahl derartiger Suchbewegungen zwischen den diversen Bedeutungsdimensionen von Religion und ihrer politischen Tragweite, vgl. zu diesen Suchbewegungen exemplarisch die Beiträge in Appel / Guanzini / Walser (Hg.) 2014; Kretschmann / Wodtke-Werner 2014; Kühnlein (Hg.) 2014; Ortner / Sabin (Hg.) 2014; Schmidt / Pitschmann (Hg.) 2014.

16 Joas 2004, S. 124.

17 Vgl. Martin 2013; Gabriel / Höhn (Hg.) 2008. Vgl ähnlich auch Asad 2003 sowie Taylor 2009. Die hier nur exemplarisch genannten kritischen Anfragen an das Postsäkularitätstheorem erheben nicht den Anspruch, die Debattenlandschaft adäquat zu repräsentieren. Im Sinne einer Minimalbestimmung des Postsäkularitätstheorems und zur Vermeidung von Missverständnissen ist jedoch festzuhalten, dass es zunächst nur um die Diagnose eines Scheiterns der Säkularisierungsthese und der damit verbundenen neuen Aufmerksamkeit für Religion, insbesondere im wissenschaftlichen und medialen Kontext, geht. Für die Anerkenntnis der bleibenden Potenziale der Religion für eine europäische Öffentlichkeit genügt zunächst dieser kleinste gemeinsame Nenner, auf den sich (wahrscheinlich) die Mehrheit der Debattenteilnehmer um die Frage nach dem Verhältnis von Säkularisierung und Demokratie einigen könnten. 
skriptiven Bedeutungsebenen des Postsäkularitätstheorems zu berücksichtigen, um die vielfältigen Missverständnisse, die mit diesem Begriff verbunden sind, zu umgehen. Unter Berücksichtigung der verschiedenen disziplinären Kontexte der Debattenbeiträge kann es gelingen, die politischen und normativen Potenziale der Religion zu analysieren und ihre Relevanz für eine europäische Öffentlichkeit zu untersuchen, ohne in die aufgeregten Debatten um eine vermeintliche „Wiederkehr der Religion" einsteigen zu müssen.

Es lässt sich also jenseits der kritischen Anfragen an das Postsäkularitätstheorem festhalten, dass gerade für eine wenig integrierte europäische Zivilgesellschaft die Religionsgemeinschaften in Europa ein mögliches einigendes Band sein können, welches, über die formale Anerkennung der Mitbürgerinnen und Mitbürger als Freie und Gleiche hinaus, weitergehende motivationale Ressourcen und solidarisches Verhalten hervorbringen kann. ${ }^{18}$ Wenn Europa den eingeschlagenen Weg der Annäherung bzw. mittelfristigen Vereinigung weitergehen möchte und nicht den derzeit aufstrebenden, meist rechtspopulistischen Re-Nationalisierungstendenzen nachgibt, dann täte es gut daran, die religiösen oder anderweitig umfassenden Identitäten seiner Bürgerinnen und Bürger nicht als reine Privatsache, sondern als öffentlich relevant einzustufen. Zugleich gilt jedoch, wie in der Einleitung bemerkt, dass Religion ein Janusgesicht hat - das Einlassen auf religiöse Potenziale kann auch durchaus gefährlich sein, wenn Religionen von solidaritätsstiftenden Lebensformen zu Instrumenten der Exklusion und der Partikularität werden.

18 Eine religionsphilosophisch höchst relevante Unterscheidung, die von Habermas vernachlässigt wird und auf die ich hier nicht weiter eingehen kann, stellt die Differenzierung der Konzepte „Religion“ und „Religiosität“ dar. Während Religion in der Regel die konkreten Überzeugungssysteme und Deutungspraxen verschiedener religiöser Gemeinschaften darstellt, ist Religiosität ein diesen Ausdeutungen zu Grunde liegendes, zunächst in seinem Aufkommen grundloses und inhaltlich nicht weiter bestimmtes Gefühl, welches jedoch als „Wurzel“ aller konkreten Religionen fungiert; vgl. exemplarisch Wendel 2006, S. 21-38; sowie Dies. 2009, S. 225-265. Es wäre anzufragen, ob die oben skizzierten Beispiele für die öffentliche Relevanz „der“ Religion nicht eher auf die motivationalen, solidaritätsstiftenden und kritischen Potenziale von „Religiosität“ abzielen, oder ob sie wirklich auf konkrete religiös-theistische Ausdeutungen von Religiosität angewiesen sind. In meinen Augen genügt zwar der Rekurs auf eine bloße Haltung im Sinne von Religiosität streng genommen nicht, um die öffentlichen Beiträge von Religionen als wertvolle Bestandteile der demokratischen Deliberation wertzuschätzen, da sie sich ja gerade durch ihre kognitive Dimension auszeichnen und im öffentlichen Diskurs kritische Anfragen stellen; jedoch wären hier weitere religionsphilosophische Präzisierungen der Konzepte von Religiosität und Religion notwendig. 


\section{Religion als gefährliches Exklusionsinstrument?}

Auch wenn Religionsgemeinschaften und religiöse Überzeugungen die exemplarisch dargelegten, heilsamen Einflüsse auf ein zusammenwachsendes Europa haben können, sollte man nicht vergessen, dass dies nur eine Seite der Medaille ist. Das Religiöse ist bleibend ambivalent, und ein Sich-Einlassen auf die Potenziale von Religion für ein vereintes Europa kann auch ein Spiel mit dem Feuer sein. Das größte Problem religiöser Überzeugungen ist, dass sie schnell von einer solidaritätsstiftenden und motivationalen Ressource in ein Instrument der Exklusion kippen können. Religiöse Überzeugungen scheinen nämlich anders strukturiert zu sein als theoretische oder moralische Überzeugungen: Es reicht nicht aus, sie auf Grundlage empirischer Belege oder vernünftiger Argumentation für wahr zu halten; vielmehr scheinen sie auch eine regulative Dimension, d.h. eine das Leben im Ganzen betreffende und die je eigene Weltsicht regulierende Funktion zu haben. In philosophischen Fachtermini gesprochen: Sie haben sowohl eine kognitiv-propositionale Dimension, d.h. eine inhaltliche Überzeugung hinsichtlich ihres Gehaltes, als auch eine regulativ-expressive Dimension, d.h. eine lebenspraktische Überzeugung hinsichtlich ihrer existenziellen Relevanz. ${ }^{19}$

Beide Aspekte, sowohl der kognitive Gehalt als auch der regulative Vollzug von religiösen Überzeugungen sind wichtige Bestandteile; keiner von ihnen kann einfach weggelassen werden. Wer nur bestimmte religiöse Sätze für wahr hält (z.B. „In Jesus Christus hat sich Gott als Liebe geoffenbart“), sich durch diese aber in keiner Weise in seiner Lebensführung beeinflussen lässt, hat ebenso wenig eine authentische religiöse Überzeugung wie jemand, der sich einem blinden Glauben hingibt und mit diesem keinerlei Überzeugung über die Beschaffenheit des Göttlichen o. ä. verbindet. Diese Doppelstruktur religiöser Überzeugungen erklärt übrigens auch, warum man allein mit vernünftigen Argumenten wohl kaum einen Atheisten zum Glauben bringen kann bzw. religiöse Menschen auch angesichts von Religionskritik, die sie zunächst nicht widerlegen können, an ihrem Glauben festhalten. Aus dieser Doppelstruktur religiöser Überzeugungen folgt, dass sie nicht den Anspruch auf allgemeine Akzeptabilität erheben können. Nur wer eine bestimmte Glaubenspraxis teilt, wird sich eine religiöse Begründung einer bestimmten Norm zu eigen machen können. Auch wenn Nicht- oder Andersgläubige religiöse Argumente inhaltlich zwar nachvollziehen können, können sie diese dennoch nicht teilen, weil ihnen die regulativ-praktische Dimension des Glaubensvollzugs fehlt.

Religionen haben damit das Potenzial, zu einem gefährlichen Instrument der Exklusion zu werden. Dies kann sich zum einen in räumlichen Trennungen äußern, wenn beispielsweise bestimmten Ländern die Zugehörigkeit zum europäi-

19 Vgl. zu dieser Strukturanalyse religiöser Überzeugungen von Stosch 2003, S. $103-$ 146. 
schen Kulturraum aufgrund religiöser Unterschiede abgesprochen wird. Hier liegt zum einen ein merkwürdig substanzialistisches Verständnis von „dem“ Christentum vor, welches gerade in Europa von unüberschaubarer Vielfalt gekennzeichnet ist, und zum anderen eine Verkennung der Komplexität historischer Prozesse, die stets zu einer Durchmischung ganz unterschiedlicher Kulturen geführt haben - die Bevölkerung Europas war nie eine homogene christliche Gemeinschaft. Zudem ist diese Form der Ethno-Religiosität durch eine problematische Vermengung von religiöser Identität und nationalen Mythen gekennzeichnet, die in ihrem radikalen Nationalismus nur schwer mit christlichen Grundüberzeugungen, wie der universal geltenden Liebe zum Nächsten, in Einklang zu bringen ist. ${ }^{20}$

Ebenso dramatisch kann Religion aber auch im (bildlich zu verstehenden) „Raum diskursiver Gründe“ als Exklusionsinstrument fungieren. Durch das Einbringen religiöser Überzeugungen in die öffentlichen Diskurse pluraler Gesellschaften könnte die Gefahr bestehen, dass politische Normen, die ja für alle gleichermaßen gelten, nicht auch für alle Betroffenen gleichermaßen gerechtfertigt sind. Wenn aber der Anspruch auf allgemeine Rechtfertigbarkeit politischer Normen aufgegeben wird, handelt man sich eine Vielzahl demokratietheoretischer Probleme ein: So ist zum einen unklar, wie eine ungerechtfertigte partikularistische Herrschaft einer weltanschaulichen Mehrheit vermieden werden kann, und zum anderen, wie man der Gefahr einer relativistischen Willkür entgehen möchte. So könnte man sich, in einem Gedankenexperiment, leicht ausmalen, dass eine religiöse Sekte die weltanschauliche Mehrheit in einer ansonsten liberalen Demokratie gewinnt und bestimmte Politiken in Zukunft auf Grundlage astrologischer Prophezeiungen oder den Geboten ihrer Gottheiten gestalten will. Einschränkungen der Freiheit aller Bürgerinnen und Bürger können auf diese Weise jedoch nicht angemessen gerechtfertigt werden, da diese so begründet werden sollten, dass ihre Rechtfertigungen von allen eingesehen und akzeptiert werden können. Dies führt viele liberale Philosophen dazu, gerade in Kontexten politischer Diskurse, die sogenannte „öffentliche Rechtfertigung“ als Bedingung der Legitimität einer demokratischen Entscheidung zu verstehen. Eine legitime demokratische Entscheidung ist darum nicht nur eine Sammlung der Vorlieben der Bürgerinnen und Bürger, sondern Ergebnis eines komplexen argumentativen Prozesses.

Exemplarisch für eine Begründung einer solchen Position kann hier John Rawls und seine Theorie des „Politischen Liberalismus“ genannt werden, in

20 Gerade in osteuropäischen Gesellschaften findet derzeit eine besorgniserregende Vermischung von Nationalstolz und religiösem Glauben statt, in denen die meist orthodoxen Staatskirchen eine enge Bindung an politisch reaktionäre Kräfte suchen und religiöse Narrative mit nationalen Gründungsmythen verknüpfen. (Vgl. exemplarisch Freeze 2013, S. 79-120.) Dieses zeitgenössische Beispiel zeigt anschaulich den möglichen Missbrauch der Religion als Instrument der Exklusion. 
welcher er unter anderem das „liberale Prinzip der Legitimität“ verteidigt. Der Grundgedanke des Prinzips besteht darin, dass eine Norm, die für alle gleichermaßen verbindlich sein soll, auch mit solchen Gründen gerechtfertigt werden muss, die für alle Betroffenen gleichermaßen einsehbar und akzeptabel sind. Eine rein partikulare Rechtfertigung einer nicht-partikularen Norm verletzt im Umkehrschluss die Freiheit und Gleichheit aller Bürger und bedroht damit die Fairness des politischen Verfahrens. Die für die Rechtfertigung allgemein verbindlicher Normen gegebenen Gründe müssen also derart sein, dass ihnen prinzipiell alle Bürger zustimmen können:

[U]nsere Ausübung politischer Macht (ist) nur dann völlig angemessen, wenn sie sich in Übereinstimmung mit einer Verfassung vollzieht, deren wesentliche Inhalte vernünftigerweise erwarten lassen, dass alle Bürger ihnen als freie und gleiche im Lichte von Grundsätzen und Idealen zustimmen, die von ihrer gemeinsamen menschlichen Vernunft anerkannt werden. ${ }^{21}$

Da religiöse Überzeugungen Rawls zufolge allerdings immer partikular sind, können sie keine Rolle in öffentlichen Rechtfertigungsdiskursen spielen - sie sollten vielmehr beschränkt werden (oder, wie er in späteren Schriften formuliert, im Notfall geäußert aber schnellstmöglich durch allgemein zugängliche Äquivalente ersetzt werden). Insbesondere die Tatsache, dass es eine Vielzahl von religiösen oder anderen umfassenden Überzeugungen über das Gute gibt, die gleichermaßen vernünftig plausibilisiert werden können, spricht dafür, angesichts dieses Faktums des vernünftigen Pluralismus auf eine allgemeine Durchsetzung der eigenen Auffassung des Guten zu verzichten. Wer also bei der Verhandlung kollektiv verbindlicher Normen religiös argumentiert, verletzt damit moralische Pflichten, die durch die Erfordernisse der öffentlichen Vernunft begründet werden-demokratische Legitimität schließe es vielmehr aus, religiöse Begründungen im öffentlichen Diskurs anzuführen.

Liberale Philosophen verdeutlichen diese Bedingungen politischer Legitimität gerne an konkreten Beispielen, um für die Intuition der Notwendigkeit reziproker und allgemein teilbarer Rechtfertigungen zu werben. So beschreibt z. B. Robert Audi eine religiöse Sekte, deren Heilige Schrift Gänseblümchen als eine heilige Spezies auszeichnet und die auf dieser Grundlage Rasenmähen unter drakonische Strafen stellen will. ${ }^{22}$ Audi zufolge könnten alle Bürgerinnen und Bürger, die nicht Mitglied dieser religiösen Sekte sind, die Begründung für ein derartiges Verbot

21 Rawls 1998, S. 223. Vgl. auch die ähnliche Formulierung in ebd., S. 318: „Als vernünftige und rationale Personen, die wissen, dass sie verschiedene vernünftige und philosophische Lehren vertreten, sollten sie [die Bürger, M. B.] in der Lage sein, die Grundlage ihres Tuns voreinander so zu erklären, dass sie vernünftigerweise mit der Zustimmung anderer rechnen können.“

22 Audi 2000, S. $93 \mathrm{f}$. 
nicht einsehen und würden sich zu Recht gegen diese illegitime Einschränkung ihrer Freiheit wehren. Umgekehrt gelte zudem, dass die Mitglieder der religiösen Sekte moralisch zu kritisieren seien, weil sie sich nicht in die Lage ihrer andersgläubigen Mitbürgerinnen und Mitbürger hineinversetzen und dementsprechend nicht erkennen, dass ihre Begründung für ein Verbot des Rasenmähens nicht allgemein teilbar ist. Wer das Prinzip der wechselseitigen Rollenübernahme bei der Rechtfertigung politischer Normen nicht beachte, läuft damit in Gefahr, eigene partikulare Wertvorstellungen zu allgemein verbindlichen Normen machen $\mathrm{zu}$ wollen. Analoges gilt, Audi zufolge, auch für Vorschriften hinsichtlich der Bekleidung oder des Verzehrs bestimmter Lebensmittel - auch hier ist eine religiöse Begründung für Einschränkungen der Freiheit aller Bürgerinnen und Bürger illegitim.

Ein zweites Beispiel wird von Robert Talisse angeführt, der die fiktive Person Stan, der ein eingefleischter Satanist ist, entwirft. ${ }^{23}$ Stan glaubt, dass die Gebote Satans allgemein verpflichtend sind und man letztlich nur dann ein erfülltes, gutes Leben führt, wenn man diesen Geboten folgt. Darum setzt er sich politisch dafür ein, eine Pflicht zur Teilnahme an satanischen Festspielen und wöchentlichen Orgien für alle verbindlich zu machen. Talisse argumentiert nun dafür, dass nicht nur die moralische Qualität des Satanismus selbst fragwürdig ist, sondern auch auf einer vorgelagerten Ebene die Moralität des Einbringens derartiger partikularer Überzeugungen in öffentliche Diskurse, die allgemein verbindliche Normen verhandeln, problematisch und illegitim ist. Weniger geht es also um eine inhaltliche Auseinandersetzung mit satanistischen Ideen, sondern um den Vorwurf, die legitimitätserzeugende Struktur öffentlicher Diskurse durch die Äußerung religiöser Überzeugungen zu verletzen. ${ }^{24}$

Allen Beispielen, die in diese Stoßrichtung von liberaler Seite angeführt werden, ist darum gemein, dass sie auf der Grundlage der fehlenden allgemeinen Akzeptierbarkeit ihrer Begründungen die Schwierigkeiten des Einbringens rein partikularer Überzeugungen illustrieren. Es ist nicht nur ein Problem, dass die vorgebrachten Begründungen merkwürdig sind (was sie zweifelsohne sind),

\section{Talisse 2012, S. 313-325.}

24 Dieses Argument ist im Übrigen eines der zentralen Argumente, das gegen einen liberalen Perfektionismus, d.h. die These der Vereinbarkeit von liberalem Staat und der Auszeichnung bestimmter Konzeptionen des guten Lebens, spricht. Der Schritt von einer Auszeichnung bestimmter Auffassungen des Guten zu einem Zwang zur Annahme dieser Auffassungen ist nicht weit, da es ja für alle Beteiligten letztlich von einem vermeintlichen Vorteil sei, diese Auffassung anzuerkennen. Dies gilt insbesondere dann, wenn man die historische Entwicklung der Anerkennung eines vernünftigen Pluralismus betrachtet - viel zu häufig wurde unter Rückgriff auf die vermeintlich bessere Kenntnis über die „eigentlichen und wahren Interessen“ von Personen Zwangsmission betrieben. Vgl. zu diesem Kritikpunkt an perfektionistischen Ansätzen die ausführliche Begriffsgeschichte des Toleranzbegriffs in Forst 2003, bes. S. 630ff. 
sondern auch, dass die Begründungen einem öffentlichen Diskurs nicht angemessen sind, da sie schon aus strukturellen Gründen nicht allgemein akzeptierbar sein können.

Gute und angemessene Begründungen von politischen Normen sind also so beschaffen, dass sie Ergebnisse der autonomen Vernunft sind und darum von jedem vernünftigen Wesen prinzipiell nachvollzogen und eingesehen werden könnten - anders ist eine begründete Abgrenzung von esoterischen Sekten und weltanschaulichen Merkwürdigkeiten kaum möglich. Diese Trennung von Religion und Politik in Europa ist eine aus der historischen Lektion der Religions- und Konfessionskriege erwachsene Errungenschaft, die nicht einfach aufgegeben werden sollte. Vielmehr kann, dies zeigen viele Beispiele gerade der europäischen Geschichte, eine derartige Vermischung von Religion und Politik desaströse Konsequenzen haben. Die entscheidende Frage ist darum, ob (und wenn ja, wie) die widerstrebenden Ergebnisse der Bedeutung von Religion für ein vereintes Europa einerseits und der Gefährdung des gesellschaftlichen Friedens durch religiöse Exklusionsmechanismen andererseits vereint werden können.

\section{Europa und seine Religionen: Ein zwiespältiges Verhältnis}

Die hier vorgelegte, religionsphilosophische Diagnose erscheint paradox: Einerseits scheint dem Projekt Europa durch religiöse Überzeugungen und Einstellungen auf Seiten seiner Bürger geholfen zu werden, um den Gefahren einer Re-Nationalisierung und dem Ende dieses friedenssichernden Projekts zu begegnen, andererseits sind die pluralistischen Gesellschaftsformen Europas nicht sonderlich kompatibel mit einem ungefilterten Eindringen religiöser Vorstellungen in die gemeinsame Politik und die demokratische Öffentlichkeit. Im Folgenden werde ich vorschlagen, zwei Sphären von Öffentlichkeit zu differenzieren, um der Doppelgesichtigkeit der Religionen begegnen zu können: die enge Sphäre der öffentlichen Rechtfertigung und die weite Sphäre der Zivilgesellschaft.

Die erste, ,enge“ Sphäre der Öffentlichkeit betrifft Diskurse, die die Rechtfertigung allgemein verbindlicher Normen thematisieren. Es ist innerhalb dieser Sphäre problematisch, bloß partikular einsehbare Gründe für allgemein gültige Normen zu geben, so dass man an einer möglichst neutralen Rechtfertigung politischer Normen festhalten und Begründungen für politische Normen, die in religiöser Sprache vorgetragen werden, äußerst vorsichtig betrachten sollte. Gerade in pluralistischen Gesellschaften ist es wichtig, dass eine gemeinsame Grundstruktur vorhanden ist, deren Vernünftigkeit von allen Gesellschaftsmitgliedern eingesehen werden kann, nicht nur von den Mitgliedern der religiösen Mehrheitskultur. Für dieses anspruchsvolle Konzept politischer Legitimität sprechen hauptsächlich zwei Gründe: erstens die Vermeidung einer ungerechtfertigten und unbegründeten Herrschaft weltanschaulicher Mehrheiten, und zweitens die (kantische) Idee des Respekts vor der Freiheit und Gleichheit aller Bürgerinnen 
und Bürger, welche nur dann wertgeschätzt wird, wenn man im öffentlichen Diskurs Begründungen anbietet, die die Kriterien der wechselseitigen Teilbarkeit und allgemeinen Zugänglichkeit erfüllen. ${ }^{25}$ Ich gehe auf beide Punkte etwas ausführlicher ein:

Wenn, erstens, eine Demokratie nicht zu einer hegemonialen Herrschaft der weltanschaulichen Mehrheit verkommen soll, ist sie auf reziprok begründete und allgemein einsehbare Rechtfertigungen für ihre Normen und Gesetze angewiesen. Andernfalls könnte eine weltanschauliche Mehrheit ihre partikularen, nicht wechselseitig akzeptablen Wertvorstellungen zu Normen machen, die auch diejenigen betreffen, die die partikular gebundenen Rechtfertigungen nicht teilen. Ein legitimer demokratischer Prozess besteht aus mehr als beispielsweise der narrativen Darstellung inkommensurabler Weltbilder, über deren Plausibilität letztlich nur durch einen Mehrheitsentscheid geurteilt wird. Der öffentliche Diskurs sollte durch den Austausch wechselseitig akzeptabler und teilbarer Gründe gekennzeichnet sein, nicht durch das bloße Teilen von Informationen oder das Äußern von Meinungen. Eine solche kognitive Entkernung demokratischer Prozesse durch die Dispensierung von Begründungspflichten hinsichtlich der im Diskurs vertretenen Geltungsansprüche würde in letzter Konsequenz zu einer non-kognitivistischen Herrschaft des Stärkeren und damit zur Abschaffung der Demokratie führen.

Jeder Diskursteilnehmer, der nicht in der Lage ist, allgemein akzeptable Gründe in Rechtfertigungsdiskursen zu geben, muss sich also den moralischen Vorwurf gefallen lassen, eigene partikulare Wertvorstellungen auf unzulässige Weise zu allgemein verbindlichen Normen überhöhen zu wollen und damit die Freiheit und Gleichheit seiner Mitbürgerinnen zu verletzen. Eine öffentliche Verhandlung politischer Normen sollte als diskursiver Prozess der Einigung auf reziprok begründete und allgemein einsehbare Konsense verstanden werden, nicht als Hegemonie einer weltanschaulichen Mehrheit, die über die reine $m a$ jority vote den jeweiligen Minderheiten ihre partikularen Wertvorstellungen als Gesetze aufzwingt. Es gilt also, dass „,dort, wo ersichtlich wird, dass man für die eigene Position, auch wenn sie die mehrheitliche ist, nur Gründe vorweisen kann, die aus Sicht der Minderheit als Übervorteilung angesehen werden können oder gar müssen, einzusehen [ist], dass diese Begründung nicht ausreicht" “. ${ }^{26}$

Der zweite, eng verwandte Grund für die Notwendigkeit reziproker Rechtfertigung ist die normative Einsicht, dass Respekt vor der Freiheit und Gleichheit aller Bürger die Pflicht zur allgemein akzeptablen Rechtfertigung der bei der Verhandlung politischer Normen vertretenen Geltungsansprüche impliziert. Der Respekt vor der formalen Freiheit und Gleichheit aller BürgerInnen gebietet es darum, eine bestimmte Art von Rechtfertigung zu liefern: Eine normativ angemessene Rechtfertigung einer allgemein verbindlichen Norm sollte für alle Be-

25 Die folgenden Aspekte beleuchte ich ausführlich in Breul 2015, S. 192-215.

26 Forst 2007, S. 223. 
troffenen gleichermaßen rational zugänglich, d.h. nachvollziehbar, und akzeptabel, d.h. wechselseitig teilbar, sein. So fordern beispielsweise Amy Gutmann und Dennis Thompson, ein Prinzip der Reziprozität in öffentlichen Diskursen als moralische Verpflichtung anzuerkennen. Der Respekt vor gleichen, freien und vernunftbegabten Teilnehmern am öffentlichen Diskurs erfordert also, allen Diskursteilnehmern Begründungen zu offerieren, die eine für alle gleichermaßen akzeptable Rechtfertigung der erhobenen Geltungsansprüche liefern können. Umgekehrt gilt dementsprechend: ,[A]ny claim fails to respect reciprocity if it imposes a requirement on other citizens to adopt one's sectarian way of life as a condition of gaining access to the moral understanding that is essential to judging the validity of one's moral claims. “27 Auch wenn Gutmann und Thompson zwar Überzeugungen über das gute Leben explizit nicht die Möglichkeit absprechen, reziprok gerechtfertigt zu werden, gilt nach der Analyse der epistemischen Struktur religiöser Überzeugungen für unseren Kontext, dass zumindest religiöse Überzeugungen dem Ideal der allgemeinen Akzeptabilität nicht gerecht werden können.

Ähnlich wie Gutmann und Thompson formuliert auch Rainer Forst die Kriterien der Reziprozität und Allgemeinheit, die geeignete Rechtfertigungen für politische Normen erfüllen sollten, da nur so der Respekt vor Mitbürgern gewahrt bleibe. ${ }^{28}$ Für Forst impliziert „,der Grundsatz, eine andere Person als ,Zweck an sich' anzuerkennen (...) ihr gegenüber Handlungen, die ihre Handlungsmöglichkeiten in moralisch relevanter Weise beschränken, reziprok-allgemein zu rechtfertigen. “29 Der normative Grund für die Akzeptanz der Kriterien von Reziprozität und Allgemeinheit liegt also in der (kantischen) Idee des Respekts vor anderen Personen als Zwecke an sich. Forst zufolge bedeutet Reziprozität, „dass niemand seinem Gegenüber bestimmte Forderungen verwehren darf, die er oder sie selbst erhebt (...), und dass niemand anderen die eigenen Wertvorstellungen und Interessen einfachhin unterstellen darf - auch nicht im Rückgriff auf ,höhere Wahrheiten“, die nicht geteilt werden“. ${ }^{30}$ Allgemeinheit heißt, dass „Gründe für allgemein geltende, grundlegende Normen unter allen Betroffenen teilbar sein müssen". ${ }^{31}$ In meinen Augen zehren beide Kriterien von der grundlegenden Einsicht, dass Begründungen für kollektiv verbindliche Normen in pluralistischen Gesellschaften erst dann legitim sind, wenn ihre Akzeptierbarkeit unabhängig vom Teilen einer bestimmten Lebenspraxis ist - letztlich sind Forsts Kriterien also zurückzuführen auf die Notwendigkeit allgemeiner Akzeptabilität bzw. Teilbarkeit von öffentlichen Gründen.

\footnotetext{
27 Gutmann / Thompson 1996, S. 57.

28 Vgl. exemplarisch Forst 2003, S. 588-629.

29 Ebd., S. 623.

30 Forst 2007, S. 15.

31 Ebd.
} 
Zugleich sollte man allerdings festhalten, dass aus diesem Appell an religiöse Bürger, sich der Grenzen der eigenen religiösen Überzeugungen bewusst zu sein, keine Privatisierung der Religion im Sinne des Rawls'schen Politischen Liberalismus gefolgert werden kann. Auch wenn religiöse Argumente und Überzeugungen keine guten Rechtfertigungen für allgemein verbindliche Gesetze und Normen sind, weil sie in pluralistischen Gesellschaften nicht von allen gleichermaßen teilbar sind, heißt das nicht, dass sie keinerlei Beitrag für die demokratische Öffentlichkeit leisten können. Vielmehr sind sie wertvolle Ressourcen der Motivation zu moralischem Handeln bzw. zivilgesellschaftlichem Engagement und liefern Anstöße zu öffentlichen Debatten, die in der latent verarmten Sprache säkularer Demokratien sonst nicht in dieser Form stattfinden würden. Wenn die politische Öffentlichkeit eine kritische Instanz sein soll, die um ebendieser kritischen Funktion willen möglichst inklusiv gestaltet werden sollte, dann wäre es leichtsinnig, wenn eine liberale Demokratie die kritischen Beiträge von religiösen Gemeinschaften oder Individuen aufgrund ihrer religiösen Herkunft a priori vom öffentlichen Diskurs ausschließen würde. Eine strikte Privatisierungsforderung ist nicht nur aufgrund ihrer diskriminatorischen Strukturierung des öffentlichen Diskurses moralisch fragwürdig, sondern auch aus dem Grund abzulehnen, dass das gefährdete Projekt der Moderne - Aufklärung, Emanzipation, Autonomie von einer diskursiven Partnerschaft mit religiösen Potenzialen profitieren kann.

So lässt sich eine zweite, umfassendere Sphäre der politischen Öffentlichkeit rekonstruieren, die man auch als zivilgesellschaftliche Sphäre bezeichnen kann. Innerhalb dieser Sphäre können die kritischen Potenziale religiöser Überlieferungen als öffentlich relevante Überzeugungen ernst genommen werden und ihre Wirkung entfalten, ohne die in einer pluralistischen Demokratie notwendige Rechtfertigungsneutralität aufzugeben bzw. den Vorrang eines autonomen und allen Betroffenen potenziell einsichtigen demokratischen Verfahrens zu gefährden. Dieser Vorschlag ähnelt dem Konzept der „civil society“ des eingangs erwähnten José Casanova, der ebenfalls einen Rückfall der Religionen hinter die Errungenschaften einer funktionalen Ausdifferenzierung ablehnt und zugleich einer liberalen Privatisierungsforderung skeptisch gegenüber steht. Casanova betont, dass Religionen sich in öffentlichen Streitfragen produktiv einbringen können und sich zugleich nicht auf die überfordernde Rolle einer die Gesellschaft im Ganzen integrierenden Gemeinschaft einlassen müssen. Gegen eine Privatisierungsforderung können Religionen auf der zivilgesellschaftlichen Ebene der politischen Öffentlichkeit „mit Nachdruck eine öffentliche politische Rolle einnehmen, ohne direkt auf die politische Gesetzgebung durchgreifen zu wollen und zu können““. ${ }^{32}$ Auf der Ebene der „,ivil society“ ist es demnach möglich, anhand eines transparenten Bündels diskursiver Kriterien die geltungstheoretische Reichweite verschiedener religiöser Überzeugungen einzuschätzen und in intersubjektiver Perspektive zu evaluieren, so dass sie die oben skizzierten positiven

32 Große Kracht 2014, S. 121. 
Einflüsse für europäische Gesellschaften hinsichtlich der Motivation zu demokratischem Engagement, der Stiftung von nationenübergreifender Solidarität und der Kritik eines entfesselten globalisierten Kapitalismus haben können. ${ }^{33} \mathrm{Zu}-$ gleich fällt man durch diese Differenzierung nicht in das andere Extrem eines kriterienlosen Inklusivismus, der sich kaum der oben skizzierten Gefahren der Willkürherrschaft weltanschaulicher Mehrheiten und des mangelnden Respekts vor Freien und Gleichen entziehen kann.

Religiöse Überlieferungen und Überzeugungen können also eine Vielzahl wichtiger Funktionen jenseits der Rechtfertigung politischer Normen übernehmen. Eine liberale Privatisierungsforderung für religiöse Überzeugungen ist darum ebenso falsch wie die Forderung nach ihrer kriterienlosen Verfugung in Rechtfertigungsdiskurse. Während die liberale Privatisierungsforderung nicht in der Lage ist, die kognitiven Aspekte und kritischen Potenziale religiöser Überzeugungen als diskursfähige und öffentlich relevante Gehalte ernst zu nehmen, vernachlässigt die Gegenposition der kriterienlosen Inklusion die regulativen Elemente religiöser Überzeugungen, die ihre allgemeine und wechselseitige Teilbarkeit verunmöglichen.

Die Grenze zwischen den zwei skizzierten Sphären der Öffentlichkeit sollte im Übrigen nicht als unüberwindbar, sondern vielmehr als porös verstanden werden, da es Prozesse der Übersetzung zwischen diesen Sphären geben kann. Religiöse Überzeugungen, die nicht ausschließlich auf persönlicher religiöser Erfahrung beruhen oder andere erstpersönliche Verstehensvoraussetzungen haben, können aus zivilgesellschaftlichen Diskursen in öffentliche Rechtfertigungsdiskurse übersetzt werden und auch dort ihre argumentative Kraft entfalten, jedoch dann in einer Sprache, die allgemein akzeptable Begründungen ermöglicht. ${ }^{34}$

33 Eine auch bei Casanova ungeklärte Frage ist, ob es auch für die zivilgesellschaftliche Sphäre der Öffentlichkeit kontexttranszendente, traditionsexterne Minimalstandards der rationalen Rechtfertigung gibt, oder ob diese Standards der Beurteilung von umfassenden Überzeugungen ebenfalls Teil der diskursiven Verhandlungsmasse sind und in verschiedenen Traditionen oder „Sprachspielen“ variieren können. Anders formuliert: Die Frage ist, ob es neben dem vernünftigen Pluralismus hinsichtlich konkurrierender Auffassungen des guten Lebens auch einen vernünftigen Pluralismus hinsichtlich der diskursiven Bewertungsstandards dieser Auffassungen geben kann. Die Beantwortung dieser Frage würde den Umfang dieses Artikels sprengen; sie ist aber ein zentrales Desiderat sowohl der religionsphilosophischen als auch der theologischen und politischtheoretischen Diskursanalyse.

34 Eine analoge Unterscheidung zwischen zwei Sphären der Öffentlichkeit trifft auch Habermas 2005, S. 119-154; allerdings unterscheidet er nicht zwischen Rechtfertigungsdiskursen und zivilgesellschaftlichen Diskursen, sondern zwischen formellen und informellen Diskursen. Formelle Diskurse sind institutionalisierte Diskurse in Gerichten oder Parlamenten, informelle Diskurse sind nicht-institutionalisierte, öffentliche Debatten. M. E. ist es sinnvoller, diese Unterscheidung der Diskurse nicht wie Habermas anhand des Grades ihrer Institutionalisiertheit, sondern anhand ihres thematischen 
Es lässt sich also abschließend festhalten, dass die Ambivalenz des Religiösen durch eine diskurstheoretische Unterscheidung zweier Sphären der Öffentlichkeit in solche Bahnen gelenkt werden kann, dass die mögliche Gefährlichkeit religiöser Überzeugungen kanalisiert und ihre unbestreitbaren Potenziale für eine stabile und integrierte Zivilgesellschaft genutzt werden können.

\section{Fazit}

Der vorliegende Text hat untersucht, inwiefern Religionen legitime und sinnvolle Beiträge zu einer demokratischen Öffentlichkeit liefern können, insbesondere angesichts des europäischen Kontexts, in dem die gegenläufigen Trends einer ReNationalisierung und einer zunehmenden Entgrenzung diagnostiziert werden können. Als Ergebnis der Untersuchung lässt sich festhalten, dass religiöse Überzeugungen ein Janusgesicht haben: Einerseits stellen sie unschätzbare Ressourcen der Solidarität, moralischen Motivation und Kritik an Herrschaftsverhältnissen dar, andererseits können sie schnell in partikulare Auffassungen des Guten kippen und als Instrumente der Exklusion das friedliche Zusammenleben in pluralen Gesellschaften gefährden. Religionen bleiben demnach ambivalente Phänomene.

Dieser Ambivalenz des Religiösen kann jedoch begegnet werden, wenn man ein differenziertes Verständnis von religiösen Überzeugungen und Sphären der Öffentlichkeit entwickelt. Es ist eine falsche Alternative, religiöse Überzeugungen entweder in laizistischer Manier ins Private abdrängen zu wollen oder sie als allgemein akzeptable und universal teilbare Überzeugungen anzusehen. Wenn man zwischen der Sphären der Rechtfertigung und Sphäre der umfassenden Zivilgesellschaft unterscheidet, ergibt sich vielmehr, dass religiöse Vielfalt auch in pluralistischen Gesellschaften nicht zwingend zu einer Aufspaltung der Gesellschaft in eine Vielzahl partikularer Parallelgesellschaften führen muss, sondern vielmehr sogar zur Integration eines europäischen Gemeinwesens beitragen kann. Es ist in meinen Augen eine offene und spannende Frage, wie sich das europäische Projekt in den nächsten Jahren weiterentwickeln wird, und inwiefern Religionen Beiträge zu diesem Projekt liefern können. In jedem Fall sollte man sich im Laufe dieses Prozesses nicht auf die kulturkämpferische Alternative

Zuschnitts vorzunehmen: In nicht-institutionalisierten Rechtfertigungsdiskursen ist es z. B. ebenfalls problematisch, religiöse Überzeugungen ungefiltert anzuführen, genau wie auch in institutionalisierten Diskursen durchaus religiöse Überzeugungen, die als motivationale Gründe eingeführt werden, zulässig sein können. Zudem ist fraglich, inwiefern die Unterscheidung ,formell-informell“ eigentlich trennscharf vorgenommen werden kann. Aus diesen Gründen halte ich die hier vorgenommene Unterscheidung in rechtfertigende und zivilgesellschaftliche Diskurse für angemessener als die Habermas'sche Lösung. 
einlassen, Religionen entweder in säkularistischer Weise als Privatsache anzusehen, oder sie als problemloses politisches Fundament Europas zu betrachten.

\section{Literaturverzeichnis}

Appel, Kurt / Guanzini, Isabella / Walser, Angelika (Hg.): Europa mit oder ohne Religion? Göttingen 2014.

Asad, Talal: Formations of the Secular: Christianity, Islam, Modernity. Stanford 2003. Audi, Robert: Religious Commitment and Secular Reason. Cambridge 2000.

Breul, Martin: „Öffentliche Gründe und die ,Doktrin der Selbstbeschränkung - Eine Kritik des liberalen Legitimitätsarguments“, in: Appel, Kurt / Guanzini, Isabella / Walser, Angelika (Hg.): Europa mit oder ohne Religion? Göttingen 2014, S. 57-67.

Ders.: Religion in der politischen Öffentlichkeit. Zum Verhältnis von religiösen Überzeugungen und öffentlicher Rechtfertigung. Paderborn 2015.

Casanova, José: Europas Angst vor der Religion. Berlin 2013.

Ders.: Public Religions in the Modern World. Chicago - London 1994.

Forst, Rainer: Das Recht auf Rechtfertigung. Frankfurt am Main 2007.

Ders.: Toleranz im Konflikt. Frankfurt am Main 2003.

Freeze, Gregory L.: „Staat, Kirche und Gläubige in Russland“, in: Graf, Friedrich W. I Meier, Heinrich (Hg.): Politik und Religion. Zur Diagnose der Gegenwart. München 2013, S. 79-120.

Gabriel, Karl / Höhn, Hans-Joachim (Hg.): Religion heute - Öffentlich und Politisch. Paderborn 2008.

Große Kracht, Hermann-Josef: „Öffentliche Religionen im säkularen Staat (Casanova)“, in: Schmidt, Thomas M. / Pitschmann, Annette: Religion und Säkularisierung. Stuttgart 2014, S. 114-126.

Gutmann, Amy / Thompson, Dennis: Democracy and Disagreement. Cambridge 1996.

Habermas, Jürgen: Glauben und Wissen. Frankfurt 2001.

Ders.: Zwischen Naturalismus und Religion. Frankfurt am Main 2005.

Ders.: „Die Lebenswelt als Raum symbolisch verkörperter Gründe“, in: Ders.: Nachmetaphysisches Denken II. Berlin 2012, S. 54-76.

Ders. : „Im Sog der Technokratie. Ein Plädoyer für europäische Solidarität“, in: Ders.: Im Sog der Technokratie. Kleine politische Schriften XII. Berlin 2013, S. 82-111.

Joas, Hans: „Religion post-säkular? Zu einer Begriffsprägung bei Jürgen Habermas“, in: Ders.: Braucht der Mensch Religion? Freiburg im Breisgau 2004, S. 122-128.

Kretschmann, Winfried / Wodtke-Werner, Verena (Hg.): Wie viel Religion verträgt der Staat? Ostfildern 2014.

Kühnlein, Michael (Hg.): Das Politische und das Vorpolitische. Über die Wertgrundlagen der Demokratie. Baden-Baden 2014.

Martin, David: On Secularization. Towards a Revised General Theory. Aldershot 2005.

Ortner, Stefan / Sabin, Stefana (Hg.): Politik ohne Gott. Wie viel Religion verträgt unsere Demokratie? Springe 2014.

Rawls, John: Politischer Liberalismus. Frankfurt 1998.

Schmidt, Thomas M. / Pitschmann, Annette (Hg.): Religion und Säkularisierung. Ein interdisziplinäres Handbuch. Stuttgart 2014. 
Stosch, Klaus von: „Was sind religiöse Überzeugungen?“, in: Joas, Hans (Hg.), Was sind religiöse Überzeugungen? (Preisschriften des Forschungsinstituts für Philosophie Hannover I). Göttingen 2003, S. 103-146.

Talisse, Robert: „Religion, Respect and Eberle's Agapic Pacifist“, in: Philosophy and Social Criticism (38) 2012, S. 313-325.

Taylor, Charles: Ein säkulares Zeitalter. Frankfurt am Main 2009.

Wendel, Saskia: „Die religiöse Selbst- und Weltdeutung des bewussten Daseins und ihre Bedeutung für eine ,moderne Religion‘. Was der ,Postmetaphysiker` Habermas über Religion nicht zu denken wagt“, in: Wenzel, Knut / Schmidt, Thomas M. (Hg.): Moderne Religion? Theologische und religionsphilosophische Reaktionen auf Jürgen Habermas. Freiburg 2009, S. 225-265.

Dies.: „Die Wurzel der Religionen“, in: FZPhTh (53) 2006, S. 21-38. 